\title{
Avaliação do nível de ansiedade e dor de pacientes em urgências endodônticas e sua influência sobre parâmetros cardiovasculares
}

\section{Evaluation of anxiety and pain levels in patients with endodontic urgency and influence in cardiovascular parameters}

\section{Patrícia Cristine de OLIVEIRA}

Professora Titular das Disciplinas de Farmacologia e Clínica Integrada da Universidade Paulista - Brasília - DF - Brasil Coordenadora do Curso de Capacitação em Sedação Consciente com Óxido Nitroso e Oxigênio na ABO - DF

\section{Darceny ZANETTA-BARBOSA}

Professor Titular da Disciplina de CTBMF da Faculdade de Odontologia - Universidade Federal de Uberlândia - MG - Brasil

\section{Hélio José de SOUZA}

Cirurgião-Dentista - Formado pela Faculdade de Odontologia - Universidade Federal de Uberlândia - Uberlândia - MG Brasil

\section{Jonas Dantas BATISTA}

Mestrando em CTBMF pela Faculdade de Odontologia - Universidade Federal de Uberlândia - Uberlândia - MG - Brasil

\section{José RANALI}

Professor Titular do Departamento de Ciências Fisiológicas, Área de Farmacologia, Anestesiologia e Terapêutica da Faculdade de Odontologia de Piracicaba - UNICAMP - SP - Brasil

\section{Marcelo Dias Moreira de Assis COSTA}

Mestrando em CTBMF pela Faculdade de Odontologia - Universidade Federal de Uberlândia - Uberlândia - MG - Brasil

\section{Paulo César AZEVEDO}

Professor Titular da Disciplina de Endodontia da Faculdade de Odontologia - Universidade Federal de Uberlândia Uberlândia - MG - Brasil

\begin{abstract}
Resumo
O objetivo do trabalho foi avaliar a influência da dor e ansiedade sobre a frequiência cardíaca (FC) e pressão arterial (PA) de 275 voluntários em situações de urgências endodônticas, selecionados de forma aleatória, entre os pacientes que procuraram o serviço do Pronto Socorro Odontológico da Faculdade de Odontologia da Universidade Federal de Uberlândia (FOUFU). Os voluntários responderam a ficha de anamnese e a Escala de Ansiedade Dental de Corah ainda na sala de espera. A Escala de 11 Pontos em Caixa foi usada para avaliar a sensação dolorosa dos voluntários, em dois momentos: antes do atendimento, para determinar a dor com que chegaram para atendimento e, após o tratamento, para determinar se houve alteração na sensação dolorosa. A PA e FC foram avaliadas através de esfingnomanômetro com coluna de mercúrio, também em dois momentos; inicialmente, com o paciente na sala de espera e, imediatamente ao término do atendimento, com o objetivo de correlacionar o controle eficaz da dor com a variação dos parâmetros cardiovasculares avaliados. De acordo com os resultados obtidos concluímos que a dor e a ansiedade não foram capazes de alterar significativamente a pressão arterial e frequiência cardíaca.
\end{abstract}

\section{Palavras-chave}

Ansiedade; dor; pressão arterial; freqüência cardíaca; odontologia. 
AVALIAÇÃO DO NÍVEL DE ANSIEDADE E DOR DE PACIENTES EM URGÊNCIAS ENDODÔNTICAS E SUA INFLUÊNCIA SOBRE PARÂMETROS CARDIOVASCULARES

\section{INTRODUÇÃO}

A ansiedade e a dor desencadeadas frente ao tratamento odontológico são fatores que podem provocar alterações comportamentais e fisiológicas, representando risco ao indivíduo e à visita ao dentista ${ }^{28}$. Dentre as possíveis conseqüências, as alterações do sistema cardiovascular, com aumento da pressão arterial e da freqüência cardíaca, são as mais relevantes principalmente nos pacientes com doenças sistêmicas ${ }^{2}, 19$.

A dor é uma experiência desagradável associada ao dano tecidual ${ }^{22}$, porém também é uma reação subjetiva que pode ser influenciada por questões culturais, afetivas, gênero e idade do indivíduo ${ }^{13,15}$. A ansiedade está intimamente relacionada a uma experiência negativa prévia ${ }^{14,16}$, novidade, incerteza, expectativa e medo $^{4,24}$. No tratamento odontológico, as mulheres mostram-se mais ansiosas que os homens ${ }^{7}$ e dentre os tratamentos odontológicos, a exodontia seguida do uso de aparelho de alta-rotação são os procedimentos que causam mais medo entre as pessoas ${ }^{1}$.

A dor e a ansiedade podem iniciar um ciclo vicioso, pois com o aumento da ansiedade, a atividade simpática é acentuada promovendo aumento da liberação de adrenalina, que ativa os nociceptores aumentando a sensibilidade dolorosa ${ }^{22}$. Nessas situações a liberação de catecolaminas, pelo sistema nervoso autônomo, induz contração das artérias, aumentando a pressão arterial e elevação na freqüência cardíaca ${ }^{6,7,29}$.

No atendimento de urgências odontológicas, a procura do paciente é motivada, na maioria das vezes, por queixa de dor ${ }^{14}$. Sendo assim, o presente estudo avaliou a correlação entre dor, ansiedade e alterações cardiovasculares em pacientes em situações de urgência odontológica.

\section{Material e método}

A execução deste estudo foi aprovada previamente pelo Comitê de Ética em pesquisa da Universidade Federal de Uberlândia sob processo número 100/04.

Foram avaliados 275 voluntários, entre os pacientes que compareceram ao Pronto Socorro Odontoló- gico da Faculdade de Odontologia da Universidade Federal de Uberlândia (FOUFU), independentemente de raça, gênero, idade, uso de medicamentos e presença de alteração sistêmica. $\mathrm{O}$ critério de inclusão dos voluntários foi a situação de urgência endodôntica. Os critérios de exclusão foram a presença de deficiência visual, auditiva ou mental, devido à impossibilidade de responder adequadamente aos questionários e escala aplicados.

Com o objetivo de caracterizar a amostra, foi feita a anamnese detalhada de cada paciente e os que concordaram com sua participação na pesquisa assinaram um termo de consentimento livre e esclarecido, conforme resolução 196/96 do Conselho Nacional de Ética em Pesquisa do Ministério da Saúde

Para avaliar o grau de ansiedade dos voluntários frente ao quadro de urgência e tratamento odontológico, utilizou-se a Escala de Ansiedade Dental de $\operatorname{Corah}^{9}$ (EADC), aplicada antes do atendimento, com o paciente ainda na sala de espera. Esta escala consiste em quatro perguntas que caracterizam o grau de ansiedade do paciente frente às diferentes situações que os fazem procurar atendimento odontológico.

A Escala de 11 Pontos em Caixa (EC) foi utilizada para quantificar a sensação dolorosa que o paciente se encontrava antes do atendimento e ao final do mesmo (Figura 1). A EC é caracterizada por 11 números escritos dentro de caixas variando de 0 a 10 , e o voluntário foi orientado de que o zero representava ausência de dor e o 10 a pior dor possível, e ele deveria marcar um "X" na caixa que contivesse o número que expressasse sua dor naquele momento.

Foram avaliados os sinais vitais de pressão arterial (PA), sistólica (PAS) e diastólica (PAD), e frequiência cardíaca (FC) dos voluntários, antes e depois do atendimento. A FC foi mensurada pelo pulso do voluntário, através de contagem tradicional por 60 segundos, e a aferição da PA, através de esfingnomanômetro com coluna de mercúrio, em 2 momentos: Antes, estando o paciente ainda na sala de espera, caracterizando o estado do paciente ao chegar no plantão; e Imediatamente após o atendimento, com o objetivo correlacionar o controle eficaz da dor com a variação da FC e PA. Todos os procedimentos foram realizados por um único avaliador

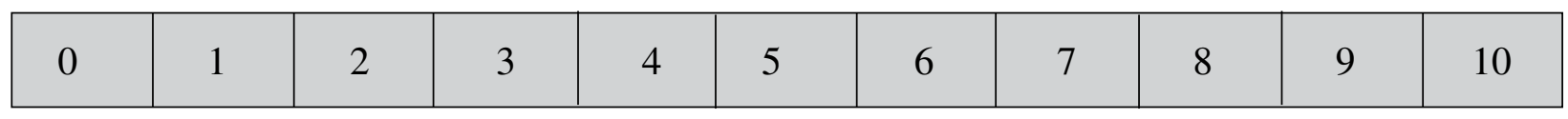

FIGURA 01 - Escala de 11 pontos em caixa (EC) 
AVALIAÇÃO DO NÍVEL DE ANSIEDADE E DOR DE PACIENTES EM URGÊNCIAS ENDODÔNTICAS E SUA INFLUÊNCIA SOBRE PARÂMETROS CARDIOVASCULARES

treinado que não realizou nenhum tipo de intervenção no atendimento odontológico desses voluntários.

Com o objetivo de verificar a existência ou não de diferenças significantes entre as medidas de PAS, PAD, FC e sensibilidade dolorosa, antes e imediatamente após o atendimento, foi aplicado o teste t de Student, com nível de probabilidade de 5\%. Para avaliar a correlação entre os dados obtidos de grau de ansiedade, sensação dolorosa, idade, FC, PAS, PAD, aplicou-se o coeficiente de correlação de Pearson, $\alpha=0,05$ (Quadro 1).

\section{Resultados}

A amostra foi constituída por 99 voluntários do sexo masculino, na faixa etária de 13 a 72 anos (idade média de 33,97 anos), e 162 do sexo feminino, de 14 a 69 anos (idade média de 32,83 anos), sendo que em relação ao grau de ansiedade a maioria apresentou-se moderadamente ansiosa (Figura 2).

Os voluntários apresentaram valores de sensação dolorosa de 6,20 antes do atendimento (valor mínimo de 1 e máximo de 10) e de 0,87 depois da intervenção (valor mínimo de 0 e máximo de 10), apresentando, portanto diferença estatisticamente significante $(\mathrm{p}=$ $0,000)$, mostrando que o controle da dor foi efetivo na maioria dos casos.

Ao se comparar os valores obtidos para as variáveis de PAS e PAD (Figura 03) e FC (Figura 4) nos períodos antes (PAS: 119,963 \pm 17,874; PAD: 79, 110 $\pm 12,071$; FC: $76,037 \pm 11,976)$ e imediatamente após o atendimento odontológico (PAS: 119,414 \pm 19,918; PAD: 79,700 \pm 13,237; FC: 75,088 $\pm 11,142$ ), verificou-se que os valores obtidos antes do tratamento foram as mais elevados, porém sem diferença estatisticamente significante (PAS: $\mathrm{p}=0,42 ; \mathrm{PAD}: \mathrm{p}=0,34$; FC: $\mathrm{p}=0,06)$.

Houve correlação positiva e significante entre sensação dolorosa e PAS $(r=0,21 ; p=0,000)$, sensação dolorosa e PAD ( $r=0,21 ; p=0,000)$, no período imediatamente após o atendimento odontológico. Encontrouse ainda correlação significante, porém negativa, entre as variáveis, como pode ser observado no Quadro 01.

Observou-se que as mulheres mostraram-se mais ansiosas (correlação positiva significante: $\mathrm{x}^{2}=5,75 ; \mathrm{C}=$ $0,14)$ e com mais dor antes do tratamento (correlação positiva significante: $\mathrm{x}^{2}=4,12 ; \mathrm{C}=0,12$ ), ambos os valores de $\mathrm{C}$ indicam $\mathrm{p}<0,05$.

\section{Dıscussão}

Sabe-se que em função do medo e da ansiedade muitas pessoas evitam o tratamento odontológico e comprometem a saúde bucal. Somente quando há agravamento destes problemas com quadros de urgências odontológicas, como os casos de pulpites e abscessos periapicais ou periodontais, por exemplo, é que estes pacientes se submetem ao tratamento, geralmente mais complexo e doloroso, envolvendo terapêutica medicamentosa para auxiliar no controle da infecção, reforçando ainda mais os sentimentos de medo e ansiedade ${ }^{3-7,13,19,20,22,29}$. Neste estudo os voluntários apresentaram-se ao pronto socorro com nível de dor maior que após a intervenção, concordando com os relatos da literatura ${ }^{3-7,13,19,20,22,29}$.

Além disso, os voluntários foram classificados, em sua maioria, como moderadamente ansiosos,

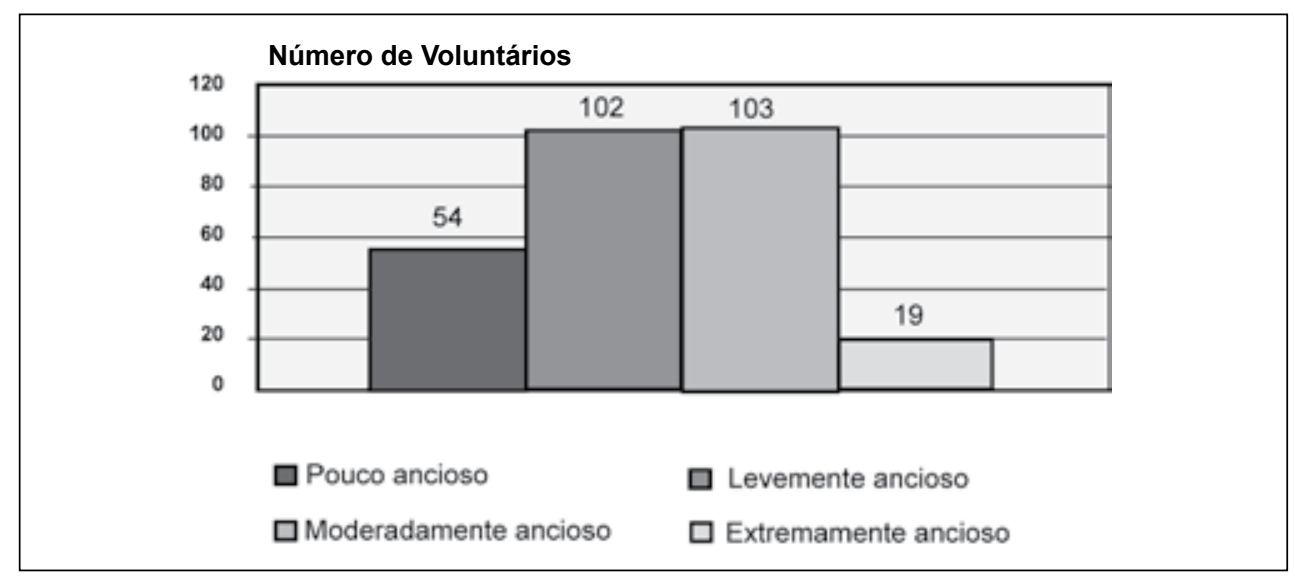

FIGURA 02 - Classificação dos voluntários quanto ao grau de ansiedade. 
Oliveira PC, Barbosa DZ, Souza HJ, Batista JD, Ranali J, Costa MDMA, Azevedo PC

AVALIAÇÃO DO NÍVEL DE ANSIEDADE E DOR DE PACIENTES EM URGÊNCIAS ENDODÔNTICAS E SUA INFLUÊNCIA SOBRE PARÂMETROS CARDIOVASCULARES

QUADRO 01 - Valores de correlação obtidos com as variáveis do estudo (dor, ansiedade, PAS, PAD e FC) nos momentos pré e pós-atendimento.

\begin{tabular}{l|c|c}
\hline Variáveis Analisadas & Valores de $\mathbf{r}$ & Probabilidades \\
\hline Dor pré-atendimento x Ansiedade & 0,0862 & 0,150 \\
\hline Dor pré-atendimento x PAS pré & 0,0403 & 0,502 \\
\hline Dor pré-atendimento x PAD pré & 0,0530 & 0,377 \\
\hline Dor pré-atendimento x FC pré & 0,0810 & 0,173 \\
\hline Ansiedade x PAS pré & 0,0063 & 0,916 \\
\hline Ansiedade x PAD pré & $-0,0424$ & 0,479 \\
\hline Ansiedade x FC pré & 0,0476 & 0,428 \\
\hline Ansiedade x Idade & $-0,2291$ & $0,000^{*}$ \\
\hline Dor pós x PAS pós & 0,2104 & $0,000^{*}$ \\
\hline Dor pós x PAD pós & 0,2097 & $0,000^{*}$ \\
\hline Dor pós x FC pós & 0,0864 & 0,150 \\
\hline Dor pós x Idade & $-0,1026$ & 0,087 \\
\hline
\end{tabular}

$\left(^{\star}\right) p<0,05$

concordando com resultados obtidos na literatura ${ }^{17}$, 18, 26, 27 de aumento da ansiedade em procedimentos mais invasivos como nas urgências odontológicas. De fato, pacientes extremamente ansiosos geralmente são vistos em serviços de urgência ${ }^{14}$ e não em serviços ambulatoriais. Também foi relatado que quanto maior a ansiedade do paciente, maior a recusa pelo tratamento odontológico e pior o índice de saúde bucal ${ }^{11,28,30}$. Avaliando-se a prevalência de medo em relação ao tratamento odontológico, concluíram que o medo só é enfrentado quando a dor causada pelo problema bucal é intensa o suficiente para minimizar a dor sentida durante os procedimentos ${ }^{25}$.

Doerr et al. ${ }^{10}$ avaliaram 445 pacientes quanto à prevalência de ansiedade frente ao tratamento odontológico, através da EADC. Dos avaliados, $10 \%$ mostraram-se ansiosos e quanto maior o grau de ansiedade, menor o índice de saúde oral. E quanto maior a procura pelo tratamento odontológico, menor o grau de ansiedade.

Clinicamente foi possível observar que as mulheres apresentaram-se mais ansiosas que os homens, concordando com vários outros relatos ${ }^{14,23,26}$. De fato, Freeeman ${ }^{12}$ relatou que a ansiedade frente a tratamentos odontológicos também tem influência de outros fatores como importância psicológica da cavidade bucal, idade, gênero do paciente e expectativa de dor, observando que as mulheres são mais ansiosas e se consideram mais medrosas em relação ao tratamento odontológico que os homens.

A EADC é um dos mais utilizados para avaliação da ansiedade ao tratamento odontológico, pois é de aplicação rápida e fácil e proporciona dados satisfatórios e confiáveis ${ }^{9,21}$. A dor, por outro lado, é difícil de ser mensurada, mas o uso da Escala de 11 pontos em Caixa apresenta menor número de erros no preenchimento por parte dos pacientes de menor nível cultural ${ }^{8}$.

A dor é uma sensação subjetiva que está vinculada a condições inerentes ao paciente, como: estado de saúde geral, idade, esclarecimento sobre o ato e a ansiedade, que podem alterar o limiar da dor do paciente ${ }^{28}$. Procedimentos clínicos mais invasivos e a punção com a agulha anestésica são sabidamente mais estressantes ${ }^{19}$.

Neste estudo, pode-se dizer que a dor antes do tratamento não influenciou de forma significativa a PA e FC dos voluntários, provavelmente pelo fato 


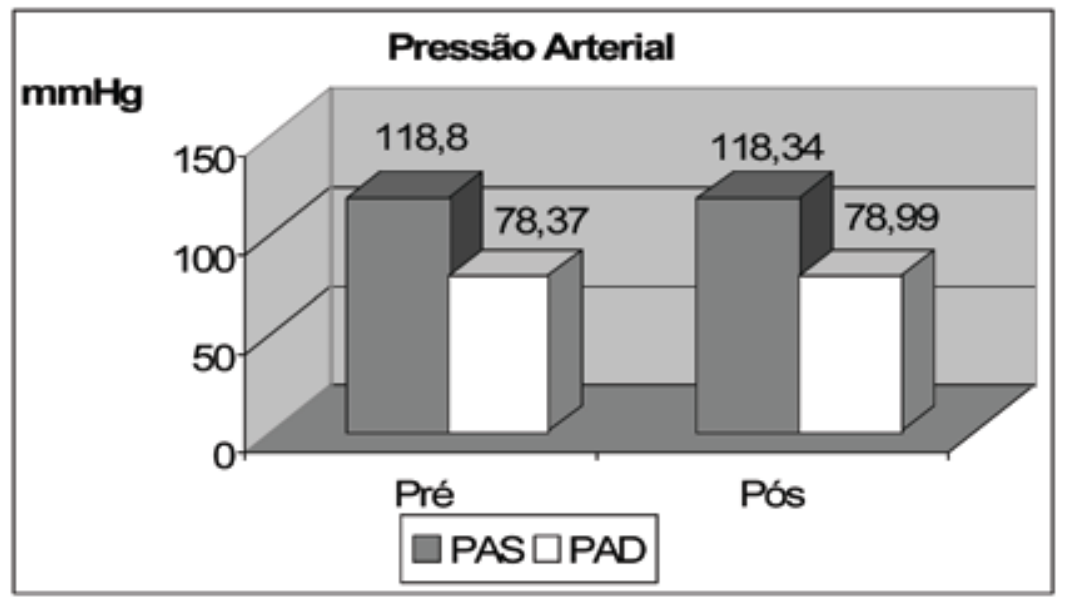

FIGURA 03 - Valores médios de PAS e PAD (em mmHg) em função do período de atendimento.

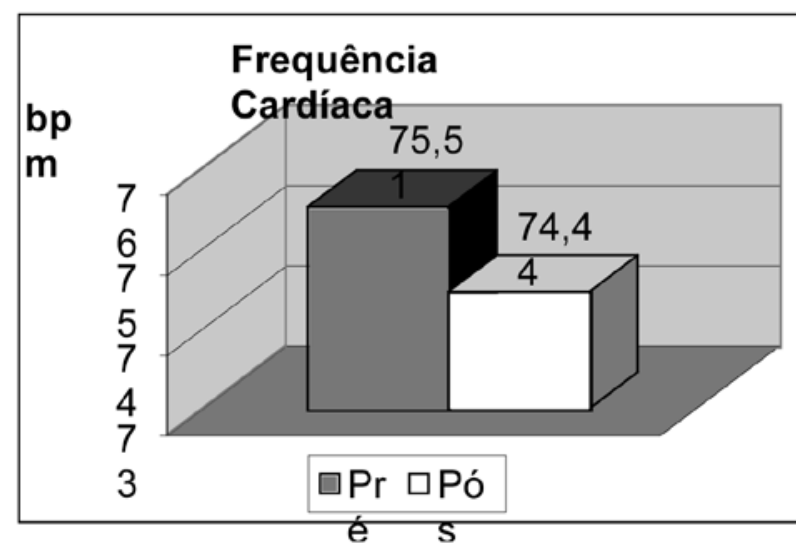

FIGURA 04 - Valores médios de FC por minuto (bpm) em função do período de atendimento.
QUADRO 02 - Valores de Ansiedade, Escala de Dor, PA e FC (média e desvio padrão).

\begin{tabular}{|c|c|}
\hline Escala de Ansiedade & $9,802 \pm 3,767$ \\
\hline Escala de Dor - Pré & $6,209 \pm 2,610^{\star}$ \\
\hline Escala de Dor - Pós & $0,886 \pm 1,966^{\star}$ \\
\hline PA. S Pré & $119,963 \pm 17,874$ \\
\hline PA. S Pós & $119,414 \pm 19,918$ \\
\hline PA. D Pré & $79,110 \pm 12,071$ \\
\hline PA. D Pós & $79,700 \pm 13,237$ \\
\hline F.C. Pré & $76,037 \pm 11,976$ \\
\hline F. C. Pós & $75,088 \pm 11,142$ \\
\hline
\end{tabular}

*Diferenças estatisticamente significantes $(\mathrm{p}=0,000)$ da sensação dolorosa não ter atingido valores extremamente significativos, embora os maiores valores tenham sido obtidos no pré-operatório ${ }^{6,7}$. Porém, observou-se voluntários com altos níveis de dor e PA elevada antes do tratamento com diminuição destes parâmetros após o atendimento.

Pode-se afirmar, portanto, que neste estudo o grau de ansiedade e dor não influenciaram significativamente os parâmetros de PA e FC e que o controle da dor através do atendimento odontológico foi fundamental para a diminuição da sensação dolorosa.

\section{Conclusão}

Dentro das condições deste estudo pode-se concluir que:

1. Os voluntários apresentaram menor dor depois do atendimento odontológico, demonstrando que seus quadros de urgências odontológicas foram satisfeitos;

2. A maioria dos voluntários na situação de urgências odontológicas mostrou-se moderadamente ansiosa;

3. A dor e a ansiedade não foram suficientes para induzir aumento de pressão arterial e frequiência cardíaca. 


\begin{abstract}
This study evaluated the influence of pain and anxiety on heart rate and blood pressure of 275 volunteers in endodontic urgency, randomly selected in dental urgency service of Dentistry Faculty of Uberlandia Federal University. The Corah's dental anxiety was applied in waiting room and 11-point box scale evaluated the painful sensibility before and after dental treatment. The same as the blood pressure and heart rate were evaluated before and immediately after the procedure, to correlate pain control and cardiovascular parameters. We concluded that pain and anxiety cannot induce blood pressure and heart rate increase.
\end{abstract}

\title{
UNITERMS
}

Anxiety; pain; heart hate; blood pressure; dentistry.

\section{RefERÊNCIAS}

1. Aartman IH. Reliability and validity of the short version of the Dental Anxiety Inventory. Community Dent Oral Epidemiol. 1998 Oct;26(5):350-4.

2. Abraham-Inpijn L, Borgmeijer-Hoelen A, Gortzak RA. Changes in blood pressure, heart rate, and electrocardiogram during dental treatment with use of local anesthesia. J Am Dent Assoc. 1988; 116 (4): 531-6.

3. Andrade ED. Terapêutica medicamentosa em odontologia: procedimentos clínicos e uso de medicamentos nas principais situações da prática odontológica. $2^{\mathrm{a}}$ ed. São Paulo: Artes Médicas; 2006.

4. Arora R. Influence of pain-free dentistry and convenience of dental office on the choice of a dental practitioner: an experimental investigation. Health Mark Q. 1999; 16 (3): 43-54.

5. Berggren U, Meynert G. Dental fear and avoidance: causes, symptoms, and consequences. J Am Dent Assoc. 1984; 109 (2): 247-51.

6. Brand HS, Abraham-Inpijn L. Cardiovascular responses induced by dental treatment. Eur J Oral Sci. 1996; 104 (3): 245-52.

7. Brand HS, Gortzak RA, Palmer-Bouva CC, Abraham RE, AbrahamInpijn L. Cardiovascular and neuroendocrine responses during acute stress induced by different types of dental treatment. Int Dent J. 1995; 45 (1): 45-8.

8. Brunetto PC. Avaliação da intensidade de dor em anestesia local: comparação entre escalas. Pesq Odontol Bras. 2003; 17 (2): 87.

9. Corah NL, Gale EN, Illig SJ. Assessment of a dental anxiety scale. J Am Dent Assoc. 1978 Nov;97(5):816-9.

10. Doerr PA, Lang WP, Nyquist LV, Ronis DL. Factors associated with dental anxiety. J Am Dent Assoc. 1998 Aug;129(8):1111-9.

11. Eitner S, Wichmann M, Paulsen A, Holst S. Dental anxiety--an epidemiological study on its clinical correlation and effects on oral health. J Oral Rehabil. 2006 Aug;33(8):588-93.

12. Freeman RE. Dental anxiety: a multifactorial aetiology. Br Dent J. 1985 Dec 21;159(12):406-8.

13. Jerjes W, Hopper C, Kumar M, Upile T, Madland G, Newman S, Feinmann C. Psychological intervention in acute dental pain: review. Br Dent J. 2007 Mar 24;202(6):337-43.

14. Kanegane K, Penha SS, Borsatti MA, Rocha RG. Ansiedade ao Tratamento odontológico em atendimentos de urgência. Rev Saude Publica. 2003 Dec;37(6):786-92. Epub 2003 Nov 27.

15. Kremer E, Atkinson JH, Ignelzi RJ. Measurement of pain: patient preference does not confound pain measurement. Pain. 1981; 10 (2): 241-8.

16. Liau FL, Kok SH, Lee JJ, Kuo RC, Hwang CR, Yang PJ. Et al. Cardiovascular influence of dental anxiety during local anesthesia for tooth extraction. Oral Surg Oral Med Oral Pathol Oral Radiol Endod. 2007 Jul 24; [Epub ahead of print]

17. Maggirias J, Locker D. Five-year incidence of dental anxiety in an adult population. Community Dent Health. 2002; 19 (3): 173-9.
18. Mamiya K, Manabe M, Aono J, Ueno H. Blood pressure control during eye surgery under local anesthesia using pulse oximetry. Ophthalmologica. 1998; 212 (4): 236-8.

19. Meyer FU. Haemodynamic changes under emotional stress following a minor surgical procedure under local anaesthesia. Int J Oral Maxillofac Surg. 1987; 16 (6): 688-94.

20. Milgrom P, Weinstein P. Dental fears in general practice: new guidelines for assessment and treatment. Int Dent J. 1993; 43 (3 Suppl 1): 288-93.

21. Newton JT, Buck DJ. Anxiety and pain measures in dentistry: a guide to their quality and application. J Am Dent Assoc. 2000 Oct;131(10):1449-57.

22. Pereira LHMC, Ramos DLP, Crosato E. Ansiedade e dor em odontologia - Enfoque psicofisiopatológico. Rev Assoc Paul Cir Dent 1995; 49 (4): 285-90.

23. Peretz B, Moshonov J. Dental anxiety among patients undergoing endodontic treatment. J Endod. 1998; 24 (6): 435-7.

24. Poulton R, Thomson WM, Brown RH, Silva PA. Dental fear with and without blood-injection fear: implications for dental health and clinical practice. Behav Res Ther. 1998; 36 (6): 591-7.

25. Quteish Taani DS. Dental fear among a young adult Saudian population. Int Dent J. 2001; 51 (2): 62-6.

26. Smyth JS. Some problems of dental treatment. Part 1. Patient anxiety: some correlates and sex differences. Aust Dent J. 1993; 38 (5): 354-9.

27. Tsuchihashi T, Takata Y, Kurokawa H, Miura K, Maruoka Y, Kajiyama $\mathrm{M}$, et al. Blood pressure response during dental surgery. Hypertens Res. 1996; 19 (3): 189-94.

28. Vassend $\mathrm{O}$. Anxiety, pain and discomfort associated with dental treatment. Behav Res Ther. 1993; 31 (7): 659-66.

29. van Wijk AJ, Hoogstraten J. Experience with dental pain and fear of dental pain. J Dent Res. 2005 Oct;84(10):947-50.

30. Woolgrove J, Cumberbatch G. Dental anxiety and regularity of dental attendance. J Dent. 1986; 14 (5): 209-13.

Recebido em 05/07/07 Aprovado em 18/09/07

Correspondência:

Jonas Dantas Batista

Av. Pará, $\mathrm{s} / \mathrm{n}^{\circ}$ - Campus Umuarama

38.400-902 - Uberlândia - MG

Secretaria de Cirurgia - B1. 4T - Sala 2G07 e-mail: jonasdantas@yahoo.com.br 